5 Bellomo R, Chapman M, Finfer S, Hickling K, Myburgh $J$. Low-dose dopamine in patients with early renal dysfunction: a placebo-controlled randomised trial. Australian and New Zealand Intensive Care Society (ANZICS) Clinical Trials Group. Lancet 2000; 356: $2139-43$.

\section{Accidental intravascular injection of levobupivacaine and lidocaine during the transarterial approach to the axil- lary brachial plexus}

To the Editor:

Racemic bupivacaine, when injected intravascularly, is associated with serious cardiac complications ${ }^{1}$ such as ventricular fibrillation resistant to successful resuscitation. No such serious outcome was reported hitherto with levobupivacaine. The present case reports the accidental intravascular injection of a combination of levobupivacaine and lidocaine used for axillary brachial plexus blockade.

A 35-yr-old patient was admitted to the hospital for orthopedic surgery. Following premedication with midazolam $(4 \mathrm{mg})$ and placement of all monitors, he received an axillary plexus block by the transarterial approach using a mixture of lidocaine $2 \%(20 \mathrm{~mL})$ and levobupivacaine $0.75 \%(20 \mathrm{~mL})$. Twenty-five millilitres of the local anesthetic mixture was deposited posterior and $15 \mathrm{~mL}$ anterior to the axillary artery. Briefly after deposition and without showing signs of light central nervous system (CNS) toxicity (lightheadedness, tinnitus, metallic taste), the patient exhibited three interrupted episodes of tonic-clonic seizures, each lasting for about three seconds and eventually resulting in unconsciousness. The patient's heart rate (HR) showed a sinus tachycardia of 160 beats $\mathrm{min}^{-1}$, the blood pressure (BP) increased to $180 / 120 \mathrm{mmHg}$ and the $\mathrm{SPO}_{2}$ decreased to $40 \%$ within one minute. For seizure control, the patient was given $5 \mathrm{mg}$ of midazolam and 100 $\mathrm{mg}$ of propofol $i v$. Following mask ventilation with $100 \%$ oxygen, he was intubated and brought to the postanesthesia care unit. His vital signs stabilized with-

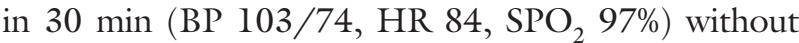
further pharmacologic support and he was extubated. Two hours after extubation, he was alert and oriented and discharged to home. The patient did not show signs of sensory and motor blockade.

A recent case report of an accidental intravascular injection following epidural anesthesia with $19 \mathrm{~mL}$ of levobupivacaine $0.75 \%$ resulted in only minor CNS side effects (drowsiness, slurred speech) and, most importantly, no cardiac sequelae. ${ }^{2}$ Plasma levels were not taken until $14 \mathrm{~min}$ after epidural injection, still they revealed a toxic range of levobupivaciane that, most likely, was substantially higher immediately after its intravascular administration. Though the severity of side effects remains unknown had racemic bupivacaine been administered in this patient, previous reports hint at a more serious outcome after racemic bupivacaine $0.75 \% .^{1}$ Similarly, no cardiac effects other than a sinus tachycardia occurred in this young and otherwise healthy patient.

It appears that levobupivacaine is a safer drug than racemic bupivacaine, still vigilance and the laws of regional anesthesia (slow and intermittent injections, frequent aspirations) need to be practiced to take advantage of levobupivacaine's wider margin of safety.

Humayon Khan MD

Peter G. Atanassoff MD

New Haven, Connecticut

\section{References}

1 Albright GA. Cardiac arrest following regional anesthesia with etidocaine or bupivacaine. Anesthesiology 1979; 51: 285-7.

2 Kopacz DJ, Allen HW. Accidental intravenous levobupivacaine. Anesth Analg 1999; 89: 1027-9.

\section{Skin analgesia with lidocaine tape prior to epidural blockade}

\section{To the Editor:}

Lidocaine tape (Penles ${ }^{\circledR}$, Japan Lederle, Tokyo, Japan) is a self-adhesive poultice for local anesthesia containing $18 \mathrm{mg}$ of lidocaine at a concentration of $60 \%$ in a $30.5 \times 50.0 \mathrm{~mm}$ polyester film. It has been reported that lidocaine tape provides effective skin analgesia, minimizing the pain caused by percutaneous cannulation, stellate ganglion block, and propofol injection. ${ }^{1-3}$ Eutectic mixture of local analgesics has also been used to alleviate cutaneous pain in children and adults. ${ }^{4}$ However, for optimal analgesic effects, the correct amount of the drug must be applied and the skin should be properly dressed for an effective absorption. ${ }^{4}$ In this regard, lidocaine tape has advantages and is frequently used because of easier application. However, although the tape is clinically useful, elevation of the pain threshold as measured by depth of needle insertion and the optimal duration of application remain unclear. 